\title{
REVISÃO INTEGRATIVA APLICADA A LEVANTAMENTOS ETNOBOTÂNICOS DE PLANTAS MEDICINAIS NO BRASIL
}

\section{INTEGRATIVE REVIEW APPLIED TO ETHNOBOTANICAL SURVEYS OF MEDICINAL PLANTS IN BRAZIL}

\author{
Camila Mabel Sganzerla ${ }^{1}$ \\ Ana Júlia Predebom ${ }^{2}$ \\ Jaqueline Veloso ${ }^{3}$ \\ Vanessa da Silva Corralo ${ }^{4}$ \\ Walter Antônio Roman Junior ${ }^{5}$
}

Submetido: 18/05/2021 / Aprovado: 01/07/2021 / Publicado: 31/10/2021.

\begin{abstract}
Resumo
Objetivamos neste estudo identificar os principais aspectos relacionados aos levantamentos etnobotânicos de plantas medicinais. Para o desenvolvimento da pesquisa foi realizada uma revisão integrativa com artigos científicos produzidos no Brasil, no período de 2010 a 2019. Foi verificado que entre os anos de 2014 e 2017 foram realizadas a maioria das investigações etnobotânicas $(64,7 \%)$, e que estes trabalhos foram desenvolvidos predominantemente na região Nordeste $(38,2 \%)$. Foi observado ainda, que o bioma mais estudado foi a Mata Atlântica (32\%), seguido da Caatinga $(26,5 \%)$ e as famílias de maior predominância foram, Asteraceae, Fabaceae e Lamiaceae. As folhas foram as partes das plantas mais usadas, e estas foram principalmente empregadas nos modos de preparo por infusão e decocção (16 e 13 citações, respectivamente). Os usos populares de plantas medicinais mais descritos estão relacionados aos tratamentos de afecções digestivas e respiratórias, seguidos de combates à inflamação e utilizadas como cicatrizantes. Estudos de revisão integrativa sobre levantamentos etnobotânicos de plantas medicinais são de grande valia para o resgate do conhecimento tradicional, para a bioprospecção racional da flora, e ainda, para ações de preservação ambiental nos diferentes biomas brasileiros.
\end{abstract}

Palavras-chave: Biomas. Conhecimento tradicional. Etnofarmacologia. Plantas bioativas.

\footnotetext{
Abstract

The aim of this study was to identify the main aspects related to the ethnobotanical surveys of medicinal plants. For the development of the investigation, an integrative review with scientific articles produced in Brazil in the period from 2010 to 2019 was used. It was found that between the

${ }^{1}$ Mestranda do Programa de Pós-Graduação em Ciências da Saúde da Universidade Comunitária da Região de Chapecó (Unochapecó). E-mail: camilamabel.203@gmail.com

${ }^{2}$ Graduanda do Curso de Farmácia da Universidade Comunitária da Região de Chapecó (Unochapecó). E-mail: predebon.ana@unochapeco.edu.br

3 Graduanda do Curso de Farmácia da Universidade Comunitária da Região de Chapecó (Unochapecó). E-mail: jaquelineveloso27@gmail.com

${ }^{4}$ Doutora em Bioquímica Toxicológica. Docente do Programa de Pós-Graduação em Ciências da Saúde Universidade Comunitária da Região de Chapecó (Unochapecó). E-mail: vcorralo@unochapeco.edu.br

${ }^{5}$ Doutor em Ciências Farmacêuticas. Docente do Programa de Pós-Graduação em Ciências da Saúde da Universidade Comunitária da Região de Chapecó (Unochapecó). E-mail: romanwa@unochapecó.edu.br 
years 2014 and 2017 most ethnobotanical investigations were carried out (64.7\%) and that these works were predominantly developed in the Northeast region (38.2\%). It was also observed that the most studied biome was Atlantic Forest (32\%), followed by "Caatinga" (26.5\%) and the most cited families were Asteraceae, Fabaceae, and Lamiaceae. The leaves were the parts of the plants most used, and these were mainly used in the modes of preparation by infusion and decoction (16 and 13 citations, respectively). The most popular uses of medicinal plants described are related to the treatment of digestive and respiratory disorders, followed by combating inflammation and used as healing agents. Integrative review applied on ethnobotanical surveys of medicinal plants is of great value for the rescue of traditional knowledge, for the rational bioprospecting of flora, and environmental preservation actions in different brazilian biomes.

Keywords: Biomes. Bioactive plants. Ethnopharmacology. Traditional knowledge.

\section{INTRODUÇÃo}

A utilização de plantas para o tratamento, cura e prevenção de doenças é uma das mais antigas formas de prática medicinal da humanidade (ŠSANTIĆ et al., 2017). Desde os tempos mais remotos, em diversas partes do mundo, há registros de enfermidades corporais ou queixas psíquicas, associadas a ação curativa de plantas. Esse fenômeno é também observado no Brasil, devido entre outros fatores, à miscigenação de vários povos principalmente os indígenas, africanos e europeus (COSTA, 2013). Essas atividades terapêuticas, muitas vezes envolvem a prática espiritual por meio de rituais que invocam forças sobrenaturais ou corporais, tais como, santidades do catolicismo popular, associado a utilização de preparados à base de plantas medicinais (GEWEHR et al., 2017; HOFFMANN-HOROCHOVSKI; ESTEVES, 2017).

Visando o resgate e a valorização deste conhecimento empírico tradicional são empregados, por exemplo, as pesquisas etnobotânicas. A etnobotânica engloba as contribuições da botânica e da etnologia e tem sido definida como um estudo capaz de compreender as inter-relações entre os seres humanos e as plantas e o modo como as plantas são utilizadas para os mais diversos fins (ALBUQUERQUE; HANAZAKI, 2006). É caracterizada como uma pesquisa científica que estuda pensamentos, crenças, sentimentos e comportamentos. Mediação de interações entre as populações humanas e os demais elementos dos ecossistemas, bem como, os impactos advindos dessa relação (ROCHA et al., 2015). Assim, estes estudos visam além do registro do uso dos recursos vegetais presentes em determinada área, conhecer as formas de manejo e como são empregadas estas matérias-primas por comunidades étnicas.

Aplicada ao estudo de plantas medicinais, a etnobotânica trabalha em estreita cumplicidade com outras disciplinas a se destacar, a etnofarmacologia que consiste em combinar informações adquiridas junto a usuários da flora medicinal, com estudos químicos e farmacológicos (ELIZABETSKY, 2003). Estes dados são importantes para o setor químico-farmacêutico, pois podem orientar pesquisas para o desenvolvimento de novas moléculas bioativas.

Nesta área do conhecimento, é consenso, devido à sua interdisciplinaridade, que os estudos envolvendo comunidades tradicionais e plantas são ordenados de acordo com o foco científico. Nessas abordagens podem ser utilizadas metodologias extremamente diversas, originárias tanto das ciências biológicas quanto sociais. Contudo, devido à crescente quantidade e à elevada complexidade de informações obtidas em diversas áreas do conhecimento, tornou-se imprescindível o desenvolvimento de artifícios capazes de delimitar etapas metodológicas mais concisas e de propiciar aos pesquisadores melhor utilização das evidências. Nesse cenário, a revisão integrativa surge como um método capaz de sintetizar os resultados obtidos em pesquisas sobre um tema ou questão, de maneira sistemática, ordenada e abrangente (SOUZA et al., 2010). 
Ainda, é importante destacar que apesar da variedade de biomas que refletem a enorme flora brasileira, aliada à grande diversidade cultural, são reduzidas as pesquisas explorando os levantamentos etnobotânicos (ALBUQUERQUE et al., 2013; APARECIDA; PAULA, 2013). Nesse contexto, este estudo pretende identificar os principais aspectos estudados nas pesquisas etnobotânicas de plantas medicinais por meio de uma revisão integrativa.

\section{MATERial E MÉtodos}

\subsection{Revisão integrativa de levantamentos etnobotânicos de planta medicinais}

Este estudo foi realizado conforme o método proposto pelo Joanna Briggs Institute (JBI, 2014). O trabalho envolveu a formulação da questão de pesquisa para a elaboração da revisão integrativa da literatura; especificação dos métodos de seleção dos estudos; procedimento de extração dos dados; análise e avaliação dos estudos incluídos na revisão integrativa da literatura; extração dos dados e apresentação da revisão/síntese do conhecimento produzido e publicado. Para tanto, foi proposta a seguinte interrogação: Quais as produções científicas sobre estudos etnobotânicos de plantas medicinais foram realizadas no Brasil, no período de janeiro de 2010 a dezembro de 2019? Para a estruturação desta questão de pesquisa, foi utilizada a estratégia PICO (JBI, 2014), que inclui: participantes; intervenção; contexto do estudo e resultados (outcomes). A coleta de dados ocorreu durante o mês de janeiro a fevereiro de 2020 nas bases de dados Literatura Latino-Americana e do Caribe em Ciências da Saúde (LILACS), Medical Literature Analysis and Retrieval Sistem on-line (Medline), Scientific Electronic Library Online (Scielo) e Pubmed inseridos na plataforma digital da BVS (Biblioteca Virtual em Saúde).

Inicialmente foi realizada uma leitura crítica e reflexiva dos títulos e dos resumos encontrados. Na sequência, foi aplicado o descritor levantamento etnobotânico e/ou ethnobotany survey, e a plataforma apresentou 688 pesquisas. Para estes trabalhos foram aplicados os seguintes critérios de inclusão: trabalhos publicados no formato de artigos científicos originais, comunicações breves e revisões sistematizadas; trabalhos disponíveis online em formato completo; estudos cujos assuntos principais foram: levantamento e/ou estudos etnobotânicos; estudos publicados no espaço temporal de 2010 a 2019; trabalhos realizados no território brasileiro; e, pesquisas realizadas nos idiomas inglês, português e espanhol, cujo título e/ou resumo, fizessem referência à temática. Desse modo, foram obtidos 56 trabalhos que, ao serem verificados individualmente quanto à qualidade metodológica, renderam 34 artigos para análise integrativa.

Para esta amostra, foi realizada uma apreciação criteriosa dos artigos selecionados, extraindo as evidências dos estudos etnobotânicos com plantas medicinais. Para melhor organização e análise, os dados foram compilados de forma descritiva em tabela, o que facilitou a identificação e a formulação de categorizações temáticas.

\section{RESUltados e DiscuSSÃo}

\subsection{Análises da revisão integrativa}

Neste estudo, realizado na Plataforma da BVS, após os critérios de análise foram selecionados 34 artigos de levantamentos etnobotânicos de plantas medicinais publicados no período de 2010 a 2019 (Tabela 1). Foi verificado que entre os anos de 2014 e 2017 foram realizadas a maioria das pesquisas etnobotânicas $(64,7 \%)$ e que estes trabalhos foram desenvolvidos em sua maioria na região Nordeste (38,2\%). Por outro lado, nos anos de 2012 e 2018 foram publicados 
somente um artigo com esta temática, nas regiões Sudeste e Norte, respectivamente. De modo interessante, durante o período analisado (2010 a 2019) foi observada uma quantidade muito reduzida de publicações realizadas nas regiões Norte e Sul (3), reconhecidas por apresentarem grande biodiversidade e comunidades étnicas.

Os biomas mais pesquisados foram a Mata Atlântica (11), Caatinga (9), seguidos do Cerrado (8), Amazônia (5) e Pantanal (1). Estes dados são semelhantes aos obtidos por Bittencourt e Paula (2012), que apontaram a Mata Atlântica (49\%), Cerrado (24\%) e Amazônia (15\%) como os principais biomas investigados. Esses resultados podem ser explicados em parte, devido ao fato do bioma Mata Atlântica se localizar mais próximo dos grandes centros de pesquisa situados principalmente na região Sudeste. Ao vincular os biomas brasileiros com as instituições onde os autores estão inseridos foi observado que em sua maioria as pesquisas correspondem ao bioma de origem. Porém, alguns estudos decorrem de parcerias realizadas com instituições de outros estados que mantêm o mesmo bioma e vinculação com instituições de ensino de outros países, como por exemplo, Itália, Canadá e Inglaterra.

É de consenso também, que pesquisas etnobotânicas envolvem um caráter multi e interdisciplinar. Nos artigos selecionados, a maioria dos trabalhos é produzida em regime de coautoria, pois, de modo geral, as investigações apresentaram entre três e cinco autores. Sem dúvida, a interação entre diversos autores e instituições em várias áreas do conhecimento pode conduzir a um caminho promissor no desenvolvimento de pesquisas com a flora brasileira (CARNEIRO et al., 2014).

Com relação aos meios de publicação, foi observado que a maioria dos artigos foi publicado na Revista Brasileira de Plantas Medicinais (49\%). No entanto, é necessário destacar que esse periódico consta como descontinuado na plataforma Scielo desde 04/2017. Na sequência surge como principal meio de divulgação de pesquisas sobre levantamentos etnobotânicos, o Journal of Ethnopharmacology, com sete trabalhos (21\%), e em seguida o Boletin Latinoamericano y del Caribe de Plantas Medicinales com quatro trabalhos (12\%). Quanto aos procedimentos metodológicos utilizados para coleta de dados foi verificada a predominância de entrevista semiestruturada (29) e a técnica de bola de neve (14), seguidas da aplicação de questionário (7).

As principais conclusões originadas das pesquisas de levantamento etnobotânico de plantas medicinais foram sobre a necessidade de preservação ambiental, o potencial de bioprospecção e o resgate do conhecimento popular (30, 28 e 26\%, respectivamente). Portanto, estudos etnobotânicos são de grande relevância na elaboração de estratégias de políticas públicas voltadas à saúde e ao meio ambiente. A descrição de dados sobre as principais famílias botânicas, partes das plantas utilizadas, formas de preparo e indicações de uso popular para os 34 artigos que compuseram esta revisão integrativa é apresentada na Tabela 2.

Neste trabalho de revisão integrativa sobre os levantamentos etnobotânicos de plantas medicinais, alguns trabalhos citaram uma única família e outros, identificaram duas ou três famílias botânicas como as predominantes. As famílias de maior abundância foram: Asteraceae (16), Fabaceae (15) e Lamiaceae (13). A família botânica Anacardiaceae e Euphorbiaceae apresentaram três citações, e as demais Caesalpiniaceae, Solanaceae, Myrtaceae, Apocynaceae, Rutaceae, somente uma citação. Com abordagem semelhante, em levantamento realizado no bioma de mata atlântica no estado de Santa Catarina, foram descritas 116 famílias, e dentre os achados a família com maior destaque foi também, a Asteraceae (119 espécies), seguida por Myrtaceae (88), Fabaceae (58) e Solanaceae (52) (GASPER et al., 2013). A grande representatividade da Asteraceae se deve ao fato que esta é uma das maiores famílias de plantas contemplando aproximadamente 1.600 gêneros e 23.000 espécies (ANDENBERG et al., 2007). Em nosso país, a família é representada por 
cerca de 180 gêneros e 1.900 espécies, distribuídas em diferentes formações vegetacionais (NAKAJIMA; SEMIR, 2001; BARROSO et al., 2007).

Quanto aos principais métodos de preparo de plantas medicinais, há destaque para a infusão (16 citações) e decocção (13 citações). Também há citações para lambedores e/ou xaropes (6) e garrafadas (3). Os preparados em forma de pó, pomada e imersão foram citados somente uma vez. Esses dados podem ser justificados em função da praticidade nos preparados caseiros obtidos por infusão e decocção, e de que estas soluções extrativas se baseiam em experiências adquiridas ao longo da vida de maneira geracional normalmente ligada a tradições e costumes socioculturais (SIQUEIRA et al., 2006; LAMBRECHTS; LALL, 2019).

Quando analisadas as partes das plantas utilizadas, foi observado que alguns estudos descreveram mais que uma parte do vegetal utilizada em seus preparados. Porém, a folha se destacou com 26 citações e as flores foram as menos utilizadas, sendo apontadas somente em um estudo. A facilidade da coleta, secagem e armazenamento das folhas, em detrimento a outras partes da planta como, raízes, cascas, frutos e inflorescências, talvez explique o maior emprego deste farmacógeno (BUENO; MARTÍNEZ; BUENO, 2016). Além disso, o uso da folha promove a conservação do recurso vegetal, pois não impede o desenvolvimento e a reprodução da planta (BELIZÁRIO; SILVA, 2012).

Os usos populares de plantas medicinais mais descritos estão relacionados aos tratamentos de afecções digestivas e respiratórias, seguidos de combate a inflamação e utilizadas como cicatrizantes. Menos citadas foram as utilizações para o tratamento das afecções renais e geniturinárias, doenças de pele, calmante e analgésica. É de consenso, que plantas com propriedades curativas, ainda se constituem como principal alternativa para a cura de doenças em comunidades tradicionais por todo o mundo (JESUS et al., 2009). Para o tratamento de doenças gastrointestinais, plantas que atuam em perturbações digestivas, hepáticas, diarreicas e disenterias têm amplo emprego, principalmente em face à carência no atendimento à saúde pública ou acesso restrito a medicamentos (GÓIS et al., 2016). Resultados análogos foram encontrados, descrevendo que em estudos etnobotânicos as doenças relacionadas aos sistemas gastrointestinal e respiratório estiveram entre as mais citadas. A elevada incidência de problemas gastrointestinais possivelmente seja devido à falta de saneamento básico, o que pode aumentar o índice de contaminação por bactérias ou verminoses (PINTO et al. 2006). Já o elevado número de indicações para afecções do sistema respiratório está associado aos fatores climáticos instáveis no Brasil (FEIJÓ et al., 2013).

Por fim, é importante destacar a relevância dos estudos etnobotânicos com plantas medicinais como estratégicos para o fortalecimento de estratégias terapêuticas. Em diversos estudos, as espécies mais citadas, além de estarem baseadas em tradições e crenças, são reconhecidas cientificamente por apresentarem efeitos farmacológicos. A Relação Nacional de Plantas Medicinais de Interesse ao SUS (RENISUS), por exemplo, é constituída por espécies vegetais contempladas em programas municipais de fitoterapia e apresentam potencial para avançar nas etapas da cadeia produtiva e de gerar produtos de interesse ao Sistema Único de Saúde (SUS) (CARNEIRO et al., 2014).

\section{CONSIDERAÇÕES FINAIS}

Apesar da vasta diversidade cultural e da enorme biodiversidade da flora brasileira, são reduzidos os estudos sobre levantamentos etnobotânicos de plantas medicinais. Visando incrementar estas pesquisas, a revisão integrativa se revela como uma estratégia fundamental pois, auxilia na verificação de dados relativos ao resgate do conhecimento tradicional, bem como, revela o potencial farmacológico das plantas bioativas nos diferentes biomas do nosso país. Além disso, é capaz de 
contribuir no fortalecimento de estratégias de inserção social contemplando não somente a bioprospecção racional, mas também, o resgate e proteção cultural, que oferecem e estabelecem ações de preservação ambiental.

\section{REFERÊNCIAS}

ALBERTASSE, P. D.; THOMAZ, L. D.; ANDRADE, M. A. Plantas medicinais e seus usos na comunidade da Barra do Jucu, Vila Velha, ES. Revista Brasileira de Plantas Medicinais, v. 12, n. 3, p. 250-260, 2010.

ALBUQUERQUE, U. P.; SILVA, J. S.; CAMPOS, J. L. A.; SOUZA, R. S.; SILVA, T. C.; ALVES, R. R. N. The current status of ethnobiological research in Latin America: Gaps and perspectives. Journal of Ethnobiology and Ethnomedicine, v. 9, n. 72, p. 1-9, 2013.

ALBUQUERQUE, U. P.; HANAZAKI, N. As pesquisas etnodirigidas na descoberta de novos fármacos de interesse médico e farmacêutico: fragilidades e perspectivas. Revista Brasileira de Farmacognosia, v. 16, p. 678-689, 2006.

ANDERBERG, A. A.; BALDWIN, B. G.; BAYER, R. G.; BREITWIESER J.; JEFFREY, C.; DILLON, M. O.; ELENAS, P.; FUNK, V.; GARCIA-JACAS, N.; HIND, D. J. N.; KARIS, P. O.; LACK, H. W.; NESOM, G.; NORDENSTAM, B.; OBERPRIELER, C.; PANERO, J. L.; PUTTOCK, C.; ROBINSON, H.; STUESSY, T.F.; SUSANNA, A.; URTUBERY, E.; VOGT, R.; WARD, J.; WATSON, L.E. Compositae. In: Flowering Plants - Eudicots: Asterales. Springer, v. 8, p. 61-588, 2007.

APARECIDA, T.; PAULA, A. Revisão Bibliométrica Sobre o Cultivo de Plantas Medicinais em Quintais Urbanos em Diferentes Regiões do Brasil (2009-2012) Journal of Health Sciences, v. 16, n. 1, p. 61-68, 2014.

BARROSO, G. M.; PEIXOTO, A. L.; COSTA, C. G.; ICHASO, C. L. F.; GUIMARÃES, E. F.; LIMA, H. C. Sistemática de Angiospermas do Brasil. v. 1. 2 ed. Viçosa. 2007. p. 310.

BELIZÁRIO, T. L.; SILVA, L. A. Abordagem etnobotânica no tratamento de parasitoses em comércios de fitoterápicos e numa comunidade rural em Uberlândia-MG. Enciclopédia Biosfera, v. 8, n. 15, p. 1730-1739, 2012.

BIESKI, I. G. C.; LEONTI, M.; ARNASON, J. T.; FERRIER, J.; RAPINSKI M.; VIOLANTE, I. M. P.; BALOGUN, S. O.; PEREIRA, J. F. C.; FIGUEIREDO, R. C. F.; LOPES, C. R. A. S.; SILVA, D. R.; PACINI, A.; ALBUQUERQUE, U. P.; MARTINS, D. T. O. Ethnobotanical study of medicinal plants by population of Valley of Juruena Region, Legal Amazon, Mato Grosso, Brazil.

Journal of Ethnopharmacology, v. 173, p. 383-423, 2015.

BITTENCOURT, L. A. F.; PAULA, A. DE. 2044-2012. Análise cienciométrica de produção científica em unidades de conservação federais do Brasil. Enciclopédia Biosfera, Científico Conhecer - Goiânia. v. 8, n. 14, p. 2044-2054, 2012.

BUENO, M. J. A.; MARTÍNEZ, B. B. BUENO, J. C. Manual utilizados na cicatrização de feridas. Univás, Pouso Alegre, 2016.

CARNEIRO, F. M.; DA SILVA, M. J. P.; BORGES, L. L.; ALBERNAZ, L, C.; COSTA, J. D. P. Tendências Dos Estudos Com Plantas Medicinais No Brasil. Revista Sapiência: sociedade, saberes e práticas educacionais - UEG, v. 3, n. 2, p. 44-75, 2014.

CARTAXO, S. L.; DE ALMEIDA SOUZA, M. M.; DE ALBUQUERQUE, U. P. Medicinal plants with bioprospecting potential used in semi-arid northeastern Brazil. Journal of Ethnopharmacology, v. 131, n. 2, p. 326-342, 2010.

CAVALHEIRO, L.; GUARIM-NETO, G. Ethnobotany and regional knowledge: Combining popular knowledge with the biotechnological potential of plants in the aldeia velha community, 
chapada dos guimarães, Mato Grosso, Brazil. Boletin Latinoamericano y del Caribe de Plantas Medicinales y Aromaticas, v. 17, n. 2, p. 197-216, 2018.

CORDEIRO, J. M. P.; FÉLIX, L. P. Conhecimento botânico medicinal sobre espécies vegetais nativas da caatinga e plantas espontâneas no agreste da paraíba, Brasil. Revista Brasileira de Plantas Medicinais, v. 16, n. 3, p. 685-692, 2014.

COSTA, P. Estudo etnobotânico de plantas antimaláricas na comunidade Céu do Mapiá, Pauini-AM. 2013. 110 p. Dissertação (Mestrado em Ciências Farmacêuticas) - Universidade Federal do Amazonas. Manaus.

COSTA, J. C.; MARINHO, M. G. V. Etnobotânica de plantas medicinais em duas comunidades do município de Picuí, Paraíba, Brasil. Revista Brasileira de Plantas Medicinais, v. 18, n. 1, p. 125134, 2016.

DA COSTA, I. B. C.; BONFIN, F. P. G.; PASA, M. C.; MONTERO, D. A. V. Ethnobotanical survey of medicinal flora in the rural community Rio dos Couros, state of Mato Grosso, Brazil.

Boletin Latinoamericano y del Caribe de Plantas Medicinales y Aromaticas, v. 16, n. 1, p. 5367, 2017.

DA SILVA MIGUÉIS, G.; DA SILVA, R. H. JÚNIOR DAMASCENO, G. A.; GUARIM-NETO, G. Plants used by the rural community of Bananal, Mato Grosso, Brazil: Aspects of popular knowledge. PLoS ONE, v. 14, n. 1, p. 1-20, 2019.

ELISABETSKY, E. Etnofarmacologia. Ciência e Cultura, Campinas, v. 55, n. 3, p. 35-36, 2003. EVANGELISTA, S. S.; SAMPAIO F. C.; PARENTE, R. C.; BANDEIRA, M. F. C. L. Fitoterápicos na odontologia: Estudo etnobotânico na cidade de Manaus. Revista Brasileira de Plantas Medicinais, v. 15, n. 4, p. 513-519, 2013.

FEIJÓ, E. V. R. S.; PEREIRA, A. S.; SOUZA, L. R.; SILVA, L. A. M.; COSTA, L. C. B. Levantamento preliminar sobre plantas medicinais utilizadas no bairro Salobrinho no município de Ilhéus, Bahia. Revista Brasileira de Plantas Medicinais, v. 15, n. 4, p. 595-604, 2013.

FREITAS, A. V. L.; COELHO, M. F. B.; PEREIRA, Y. B.; FREITAS NETO, E. C.; AZEVEDO, R. A. B. Diversidade e usos de plantas medicinais nos quintais da comunidade de São João da Várzea em Mossoró, RN. Revista Brasileira de Plantas Medicinais, v. 17, n. 4, p. 845-856, 2015. GASPER, A. L. DE.; SEVEGNANI, L.; VIBRANS, C. A.; SOBRAL, M.; UHLMANN, A.; LINGNER, D. V.; RIGON-JUNIOR, M. J.; VERDI, M.; SANTOS, A. S.; DREVECK, S.; KORTE, A. Inventário florístico florestal de Santa Catarina: espécies da Floresta Ombrófila Mista. Rodriguésia, v. 64, n. 2, p. 201-210, 2013.

GEWEHR, R. B.; BAÊTA, J.; GOMES, E.; TAVARES, R. Sobre as práticas tradicionais de cura: subjetividade e objetivação nas propostas terapêuticas contemporâneas. Psicologia USP, v. 28, n. 1, p. 33-43, 2017.

GOIS, M. A. F.; LUCAS, F. C. A.; COSTA, J. C. M.; MOURA, P. H. B. DE; LOBATO, G. DE J.M. Etnobotânica de espécies vegetais medicinais no tratamento de transtornos do sistema gastrointestinal. Revista Brasileira de Plantas Medicinais, v. 18, n. 2, p. 547-557, 2016.

GOMEZ, M.; LUCENA, E. A. R. M. DE; GOMBERG, E. Análise das publicações etnobotânicas sobre plantas medicinais da Mata Atlântica na Região Sul do Estado da Bahia, Brasil. Revista Fitos, v. 10, n. 2, p. 115-140, 2016.

GRIZ, S. A. S.; MATOS-ROCHA, T. J.; SANTOS, A. F.; COSTA, J. G.; MOUSINHO, K. C. Medicinal plants profile used by the 3rd District population of Maceió-AL. Brazilian Journal of Biology, v. 77, n. 4, p. 794-802, 2017.

HOFFMANN-HOROCHOVSKI, M. T.; ESTEVES, N. DOS S. Práticas tradicionais de cura na comunidade rural rio verde em Guaraqueçaba (PR). Divers@Revista Eletrônica Interdisciplinar, n. 2, p. 69-78, 2017. 
JESUS, N. Z. T.; LIMA, J. C. S.; DA SILVA, R. M.; ESPINOSA, M. M.; MARTINS, D. T. O. Levantamento etnobotânico de plantas popularmente utilizadas como antiúlceras e antiinflamatórias pela comunidade de Pirizal, Nossa Senhora do Livramento - MT, Brasil. Revista Brasileira de Farmacognosia, v. 19, n. 1, p. 130-139, 2009.

JOANNA BRIGGS INSTITUTE (JBI). Joanna Briggs Institute Reviewers' manual: Methodology for JBI mixed methods systematic reviews [Internet]. 2014 [cited 2020 Oct 08]. Available from: https://nursing.lsuhsc.edu/JBI/docs/ReviewersManuals/Mixed-Methods.pdf LAMBRECHTS, I. A.; LALL, N.; Plectranthus neochilus. In: Underexplored Medicinal Plants from Sub-Saharan Africa. Ed.: Elsevier, cap 36, p. 235-240, 2019.

LIMA, I. E. O.; NASCIMENTO, L. A. M.; SILVA, M. S. Comercialização de plantas medicinais no município de Arapiraca-AL. Revista Brasileira de Plantas Medicinais, v. 18, n. 2, p. 462-472, 2016.

LIPORACCI, H. S. N.; SIMÃO, D. G. Levantamento etnobotânico de plantas medicinais nos quintais do Bairro Novo Horizonte, Ituiutaba, MG. Revista Brasileira de Plantas Medicinais, v. 15, n. 4, p. 529-540, 2013.

MARINHO, M. G. V.; SILVA, C. C.; ANDRADE, L. H. C. Levantamento etnobotânico de plantas medicinais em área de caatinga no município de São José de Espinharas, Paraíba, Brasil. Revista Brasileira de Plantas Medicinais, v. 13, n. 2, p. 170-180, 2011.

MARTINS-RAMOS, D.; BORTOLUZZI, R. L. C.; MANTOVANI, A. Plantas medicinais de um remascente de Floresta Ombrófila Mista Altomontana, Urupema, Santa Catarina, Brasil. Revista Brasileira de Plantas Medicinais. v. 12, n. 3, p. 380-397, 2010.

MESQUITA, U. O.; TAVARES-MARTINS, A. C. C. Etnobotánica de plantas medicinales en la comunidad de Caruarú, Isla del Mosqueiro, Belém-PA, Brasil. Boletin Latinoamericano y del Caribe de Plantas Medicinales y Aromaticas, v. 17, n. 2, p. 130-159, 2018.

MESSIAS, M. C. T. B.; MENEGATTO, M. F.; PRADO, A. C. C.; SANTOS, B. R.; GUIMARÃES, M. F. M. Uso popular de plantas medicinais e perfil socioeconômico dos usuários: Um estudo em área urbana em Ouro Preto, MG, Brasil. Revista Brasileira de Plantas Medicinais, v. 17, n. 1, p. 76-104, 2015.

NAKAJIMA, N. J.; SEMIR, J. Asteraceae do Parque Nacional da Serra da Canastra, Minas Gerais, Brasil. Revista Brasileira de Botânica. v. 24, n. 4, p. 471- 478, 2001.

OLIVEIRA, A. K. M.; OLIVEIRA, N. A.; RESENDE U, M.; MARTINS, P. F. R. B.. Ethnobotany and traditional medicine of the inhabitants of the Pantanal Negro sub-region and the raizeiros of Miranda and Aquidauna, Mato Grosso do Sul, Brazil. Brazilian Journal of Biology, v. 71, n. 1 suppl 1, p. 283-289, 2011.

OLIVEIRA, E. R.; MENINI NETO, L. Levantamento etnobotânico de plantas medicinais utilizadas pelos moradores do povoado de Manejo, Lima Duarte - MG. Revista Brasileira de Plantas Medicinais, v. 14, n. 2, p. 311-320, 2012.

OLIVEIRA, G. L.; OLIVEIRA, A. F. M.; ANDRADE, L. DE H. C. Medicinal and toxic plants from Muribeca Alternative Health Center (Pernambuco, Brazil): An ethnopharmacology survey. Boletin Latinoamericano y del Caribe de Plantas Medicinales y Aromaticas, v. 14, n. 6, p. 470-483, 2015.

PEDROLLO, C. T.; KINUPP, V. F.; JR SHEPARD, G.; HEINRICH, M. Medicinal plants at Rio Jauaperi, Brazilian Amazon: Ethnobotanical survey and environmental conservation. Journal of Ethnopharmacology, v. 186, p. 111-124, 2016.

PENIDO, A. B.; MORAIS, S. M.; RIBEIRO, A. B.; SILVA, A. Z. Ethnobotanical study of medicinal plants in Imperatriz, State of Maranhão, Northeastern Brazil. Acta Amazonica, v. 46, n. 4, p. 345-354, 2016. 
PINTO, E. P. P.; AMOROZO, M. C. M.; FURLAN, A. Conhecimento popular sobre plantas medicinais em comunidades rurais de mata atlântica - Itacaré, BA, Brasil. Acta Botânica Brasílica, v. 20, n. 4, p. 751-762, 2006.

RIBEIRO, D. A.; DE OLIVEIRA, L. G. S.; DE MACÊDO, D. G.; DE MENEZES, I, R, A.; DA COSTA, J. G. M.; DA SILVA, M. A. P.; LACERDA, S. R.; SOUZA, M. M. A. Promising medicinal plants for bioprospection in a Cerrado area of Chapada do Araripe, Northeastern Brazil. Journal of Ethnopharmacology, v. 155, n. 3, p. 1522-1533, 2014a.

RIBEIRO, D. A.; MACÊDO, D. G.; OLIVEIRA, L. G. S.; SARAIVA, M. E.; OLIVEIRA, S. F.; SOUZA, M. M. A.; MENEZES, I. R. A. Potencial terapêutico e uso de plantas medicinais em uma área de Caatinga no estado do Ceará, nordeste do Brasil. Revista Brasileira de Plantas Medicinais, v. 16, n. 4, p. 912-930, 2014b.

RIBEIRO, R. V.; BIESKI, I. G. C.; BALOGUN, S. O.; MARTINS, D. T. O. Ethnobotanical study of medicinal plants used by ribeirinhos in the North Araguaia microregion, Mato Grosso, Brazil.

Journal of Ethnopharmacology, v. 205, n. April, p. 69-102, 2017.

ROCHA, J. A.; BOSCOLO, O. H. FERNANDES, L. R. R. M. V. Etnobotânica: um instrumento para valorização e identificação de potenciais de proteção do conhecimento tradicional. Interações (Campo Grande), v. 16, n. 1, p. 67-74, 2015.

RODRIGUES, A. P.; ANDRADE, L. H. C. Levantamento etnobotânico das plantas medicinais utilizadas pela comunidade de Inhamã, Pernambuco, Nordeste do Brasil. Revista Brasileira de Plantas Medicinais, v. 16, n. 3, p. 721-730, 2014.

ŠANTIĆ, Ž; PRAVDIĆ, N; BEVANDA, M; GALIĆ, K. The historical use of medicinal plants in traditional and scientific medicine. Psychiatria Danubina. v.5, n. 1-2, p. 69-74, 2017

SANTOS, M. O.; DE ALMEIDA, B. V.; RIBEIRO, A. D.; DE MACÊDO, D. G.; MACÊDO, M. J. F.; MACEDO, J. G. F.; DE SOUZA, F. F. S.; DE OLIVEIRA, L. G. S.; SARAIVA, M. E.; ARAÚJO, T. M.S.; SOUZA, M. M. A. The conservation of native priority medicinal plants in a caatinga area in Ceará, northeastern Brazil. Anais da Academia Brasileira de Ciências, v. 89, n. 4, p. 2675-2685, 2017.

SARAIVA, M. E.; ULISSES A. V. R.; RIBEIRO, D. A.; DE OLIVEIRA, L. G. S.; DE MACÊDO, D. G; DE SOUZA, F. F. S.; DE MENEZES, I. R. A.; SAMPAIO, E. V. S. B.; SOUZA, M. M. A. Plant species as a therapeutic resource in areas of the savanna in the state of Pernambuco, Northeast Brazil. Journal of Ethnopharmacology, v. 171, p. 141-153, 2015.

SILVA, C, G.; MARINHO, M, G, V.; LUCENA, M, F, A.; COSTA, J, G, M.; Levantamento etnobotânico de plantas medicinais em área de Caatinga na comunidade do Sítio Nazaré, município de Milagres, Ceará, Brasil. Revista Brasileira de Plantas Medicinais, v. 17, n. 1, p. 133-142, 2015. SIQUEIRA, K. M.; BARBOSA, M. A.; BRASIL, B. B.; OLIVEIRA, L. M. C.; ANDRAUS, L. M. S. Crenças populares referentes à saúde: apropriação de saberes sócio-culturais. Texto \& Contexto - Enfermagem, v. 15, n. 1, p. 68-73, 2006.

SOUZA, M. T.; SILVA, M. D., CARVALHO, R. Revisão integrativa: o que é e como fazer? Einstein. v. 8, n. 1, p. 102-6, 2010.

SOUZA, M. J. M.; MORAL, F. F.; NASCIMENTO, G. N. L.; SOARES, P. N.; AVERSIFERREIRA, T. A. Plantas medicinais usadas pela comunidade do bairro Itamaraty em Anápolis, Estado de Goiás, Brasil. Acta Scientiarum - Health Sciences, v. 32, n. 2, p. 177-184, 2010.

TOMAZI, L. B.; AGUIAR, P. A.; CITADINI -ZANETTE, V.; ROSSATO, A. E. Estudo etnobotânico das árvores medicinais do Parque Ecológico Municipal José Milanese, Criciúma, Santa Catarina, Brasil. Revista Brasileira de Plantas Medicinais, v. 16, n. 2 SUPPL. 1, p. 450-461, 2014. 
TRIBESS, B.; PINTARELLI, M, G.; BINI, L, A.; CAMARGO A.; FUNEZ, L. A.; GASPER A, L.; BENI, A. L. B. Ethnobotanical study of plants used for therapeutic purposes in the Atlantic Forest region, Southern Brazil. Journal of Ethnopharmacology, v. 164, p. 136-146, 2015.

ZUCCHI, M. R.; OLIVEIRA JÚNIOR, V. F.; GUSSONI, M. A.; SILVA, M. B.; SILVA, F. C.; MARQUES, N. E. Levantamento etnobotânico de plantas medicinais na cidade de Ipameri - GO. Revista Brasileira de Plantas Medicinais, v. 15, n. 2, p. 273-279, 2013. 
Tabela 1 - Artigos científicos de pesquisa etnobotânica envolvendo plantas medicinais, publicados no período de 2010 a 2019 na Plataforma da BVS.

\begin{tabular}{|c|c|c|c|c|}
\hline Ordem & Autores & Título da pesquisa & Publicação & $\begin{array}{c}\text { Ano de } \\
\text { publicação }\end{array}$ \\
\hline 01 & CARTAXO et al. & $\begin{array}{c}\text { Medicinal plants with bioprospecting } \\
\text { potential used in semi-arid northeastern } \\
\text { Brazil }\end{array}$ & $\begin{array}{c}\text { Journal of } \\
\text { Ethnopharmacology }\end{array}$ & 2010 \\
\hline 02 & SOUSA et al. & $\begin{array}{c}\text { Medicinal plants used by Itamaraty } \\
\text { community nearby Anápolis, Goiás State, } \\
\text { Brazil }\end{array}$ & $\begin{array}{l}\text { Acta Scientiarum - } \\
\text { Health Sciences }\end{array}$ & 2010 \\
\hline 03 & $\begin{array}{l}\text { ALBERTASSE et } \\
\text { al. }\end{array}$ & $\begin{array}{l}\text { Medicinal plants and their uses in Barra } \\
\text { do Jucu community, Vila Velha } \\
\text { Municipality, Espírito Santo State, Brazil }\end{array}$ & $\begin{array}{l}\text { Revista Brasileira de } \\
\text { Plantas Medicinais }\end{array}$ & 2010 \\
\hline 04 & $\begin{array}{l}\text { MARTINS- } \\
\text { RAMOS et al. }\end{array}$ & $\begin{array}{c}\text { Medicinal plants in a remnant of High } \\
\text { Montane Araucaria Moist Forest, } \\
\text { Urupema Municipality, Santa Catarina } \\
\text { State, Brazil }\end{array}$ & $\begin{array}{l}\text { Revista Brasileira de } \\
\text { Plantas Medicinais }\end{array}$ & 2010 \\
\hline 05 & MARINHO et al. & $\begin{array}{c}\text { Ethnobotanical survey of medicinal } \\
\text { plants in a caatinga area in São José de } \\
\text { Espinharas Municipality, Paraíba State, } \\
\text { Brazil }\end{array}$ & $\begin{array}{l}\text { Revista Brasileira de } \\
\text { Plantas Medicinais }\end{array}$ & 2011 \\
\hline 06 & OLIVEIRA et al. & $\begin{array}{l}\text { Etnobotânica e medicina tradicional dos } \\
\text { habitantes da sub-região do Pantanal } \\
\text { Negro e dos raizeiros de Miranda e } \\
\text { Aquidauna, Mato Grosso do Sul, Brasil }\end{array}$ & $\begin{array}{l}\text { Revista Brasileira de } \\
\text { Biologia }\end{array}$ & 2011 \\
\hline 07 & $\begin{array}{l}\text { OLIVEIRA; } \\
\text { MENINI-NETO }\end{array}$ & $\begin{array}{c}\text { Ethnobotanical survey of the medicinal } \\
\text { plants used by dwellers of Manejo } \\
\text { Village, Lima Duarte-Minas Gerais State, } \\
\text { Brazil }\end{array}$ & $\begin{array}{l}\text { Revista Brasileira de } \\
\text { Plantas Medicinais }\end{array}$ & 2012 \\
\hline 08 & $\begin{array}{l}\text { LIPORACCI; } \\
\text { SIMÃO }\end{array}$ & $\begin{array}{c}\text { Levantamento etnobotânico de plantas } \\
\text { medicinais nos quintais do Bairro Novo } \\
\text { Horizonte, Ituiutaba, MG }\end{array}$ & $\begin{array}{l}\text { Revista Brasileira de } \\
\text { Plantas Medicinais }\end{array}$ & 2013 \\
\hline 09 & ZUCCHI et al. & $\begin{array}{l}\text { Levantamento etnobotânico de plantas } \\
\text { medicinais na cidade de Ipameri - GO }\end{array}$ & $\begin{array}{l}\text { Revista Brasileira de } \\
\text { Plantas Medicinais }\end{array}$ & 2013 \\
\hline 10 & $\begin{array}{l}\text { EVANGELISTA } \\
\text { et al., }\end{array}$ & $\begin{array}{l}\text { Phytotherapics in Odontology: } \\
\text { ethnobotanical study in Manaus }\end{array}$ & $\begin{array}{l}\text { Revista Brasileira de } \\
\text { Plantas Medicinais }\end{array}$ & 2013 \\
\hline 11 & $\begin{array}{l}\text { RIBEIRO et al. } \\
\text { (a) }\end{array}$ & $\begin{array}{c}\text { Promising medicinal plants for } \\
\text { bioprospection in a Cerrado area of } \\
\text { Chapada do Araripe, Northeastern Brazil }\end{array}$ & $\begin{array}{c}\text { Journal of } \\
\text { Ethnopharmacology }\end{array}$ & 2014 \\
\hline 12 & $\begin{array}{l}\text { RIBEIRO et al. } \\
\text { (b) }\end{array}$ & $\begin{array}{l}\text { Potencial terapêutico e uso de plantas } \\
\text { medicinais em uma área de Caatinga no } \\
\text { estado do Ceará, nordeste do Brasil }\end{array}$ & $\begin{array}{l}\text { Revista Brasileira de } \\
\text { Plantas Medicinais }\end{array}$ & 2014 \\
\hline
\end{tabular}




\begin{tabular}{|c|c|c|c|c|}
\hline 13 & $\begin{array}{l}\text { RODRIGUES; } \\
\text { ANDRADE }\end{array}$ & $\begin{array}{l}\text { Levantamento etnobotânico das plantas } \\
\text { medicinais utilizadas pela comunidade de } \\
\text { Inhamã, Pernambuco, Nordeste do Brasil }\end{array}$ & $\begin{array}{l}\text { Revista Brasileira de } \\
\text { Plantas Medicinais }\end{array}$ & 2014 \\
\hline 14 & $\begin{array}{l}\text { CORDEIRO; } \\
\text { FÉLIX }\end{array}$ & $\begin{array}{c}\text { Botanical medical knowledge of native } \\
\text { species of the Caatinga and spontaneous } \\
\text { plants in the Agreste region of the state of } \\
\text { Paraíba, Brazil }\end{array}$ & $\begin{array}{l}\text { Revista Brasileira de } \\
\text { Plantas Medicinais }\end{array}$ & 2014 \\
\hline 15 & TOMAZI et al. & $\begin{array}{c}\text { Estudo etnobotânico das árvores } \\
\text { medicinais do Parque Ecológico } \\
\text { Municipal José Milanese, Criciúma, } \\
\text { Santa Catarina, Brasil }\end{array}$ & $\begin{array}{l}\text { Revista Brasileira de } \\
\text { Plantas Medicinais }\end{array}$ & 2014 \\
\hline 16 & SARAIVA et al. & $\begin{array}{c}\text { Plant species as a therapeutic resource in } \\
\text { areas of the savanna in the state of } \\
\text { Pernambuco, Northeast Brazil }\end{array}$ & $\begin{array}{c}\text { Journal of } \\
\text { Ethnopharmacology }\end{array}$ & 2015 \\
\hline 17 & OLIVEIRA et al. & $\begin{array}{l}\text { Medicinal and toxic plants from } \\
\text { Muribeca Alternative Health Center } \\
\text { (Pernambuco, Brazil): an } \\
\text { ethnopharmacology survey }\end{array}$ & $\begin{array}{c}\text { Boletín } \\
\text { Latinoamericano y } \\
\text { del Caribe de Plantas } \\
\text { Medicinales y } \\
\text { Aromáticas }\end{array}$ & 2015 \\
\hline 18 & TRIBESS et al. & $\begin{array}{l}\text { Ethnobotanical study of plants used for } \\
\text { therapeutic purposes in the Atlantic } \\
\text { Forest region, Southern Brazil }\end{array}$ & $\begin{array}{c}\text { Journal of } \\
\text { Ethnopharmacology }\end{array}$ & 2015 \\
\hline 19 & BIESKI et al. & $\begin{array}{c}\text { Ethnobotanical study of medicinal plants } \\
\text { by population of Valley of Juruena } \\
\text { Region, Legal Amazon, Mato Grosso, } \\
\text { Brazil }\end{array}$ & $\begin{array}{c}\text { Journal of } \\
\text { Ethnopharmacology }\end{array}$ & 2015 \\
\hline 20 & FREITAS et al. & $\begin{array}{c}\text { Diversidade e usos de plantas medicinais } \\
\text { nos quintais da comunidade de São João } \\
\text { da Várzea em Mossoró, RN }\end{array}$ & $\begin{array}{l}\text { Revista Brasileira de } \\
\text { Plantas Medicinais. }\end{array}$ & 2015 \\
\hline 21 & SILVA et al. & $\begin{array}{l}\text { Levantamento etnobotânico de plantas } \\
\text { medicinais em área de Caatinga na } \\
\text { comunidade do Sítio Nazaré, município } \\
\text { de Milagres, Ceará, Brasil }\end{array}$ & $\begin{array}{l}\text { Revista Brasileira de } \\
\text { Plantas Medicinais }\end{array}$ & 2015 \\
\hline 22 & MESSIAS et al. & $\begin{array}{l}\text { Uso popular de plantas medicinais e } \\
\text { perfil socioeconômico dos usuários: um } \\
\text { estudo em área urbana em Ouro }\end{array}$ & $\begin{array}{l}\text { Revista Brasileira de } \\
\text { Plantas Medicinais }\end{array}$ & 2015 \\
\hline 23 & $\begin{array}{l}\text { PEDROLLO } \\
\text { et al. }\end{array}$ & $\begin{array}{l}\text { Medicinal plants at Rio Jauaperi, } \\
\text { Brazilian Amazon: Ethnobotanical } \\
\text { survey and environmental conservation }\end{array}$ & $\begin{array}{c}\text { Journal of } \\
\text { Ethnopharmacology }\end{array}$ & 2016 \\
\hline 24 & $\begin{array}{l}\text { COSTA; } \\
\text { MARINHO }\end{array}$ & $\begin{array}{c}\text { Etnobotânica de plantas medicinais em } \\
\text { duas comunidades do município de Picuí, } \\
\text { Paraíba, Brasil }\end{array}$ & $\begin{array}{l}\text { Revista Brasileira de } \\
\text { Plantas Medicinais }\end{array}$ & 2016 \\
\hline 25 & LIMA et al. & $\begin{array}{c}\text { Comercialização de Plantas Medicinais } \\
\text { no Município de Arapiraca-AL }\end{array}$ & $\begin{array}{l}\text { Revista Brasileira de } \\
\text { Plantas Medicinais }\end{array}$ & 2016 \\
\hline
\end{tabular}




\begin{tabular}{|c|c|c|c|c|}
\hline 26 & PENIDO et al. & $\begin{array}{l}\text { Ethnobotanical study of medicinal plants } \\
\text { in } \\
\text { Imperatriz, State of Maranhão, } \\
\text { Northeastern Brazil }\end{array}$ & $\begin{array}{c}\text { Acta } \\
\text { Amazônica }\end{array}$ & 2016 \\
\hline 27 & GOMEZ et al. & $\begin{array}{c}\text { Análise das publicações etnobotânicas } \\
\text { sobre plantas medicinais da Mata } \\
\text { Atlântica na Região Sul do Estado da } \\
\text { Bahia, Brasil }\end{array}$ & Revista Fitos & 2016 \\
\hline 28 & RIBEIRO et al. & $\begin{array}{l}\text { Ethnobotanical study of medicinal plants } \\
\text { used by ribeirinhos in the North Araguaia } \\
\text { microregion, Mato Grosso, Brazil }\end{array}$ & $\begin{array}{c}\text { Journal of } \\
\text { Ethnopharmacology }\end{array}$ & 2017 \\
\hline 29 & GRIZ et al. & $\begin{array}{l}\text { Medicinal plants profile used by the } 3 \text { rd } \\
\text { District population of Maceió-AL }\end{array}$ & $\begin{array}{l}\text { Brazilian Journal of } \\
\text { Biology }\end{array}$ & 2017 \\
\hline 30 & SANTOS et al. & $\begin{array}{c}\text { The conservation of native priority } \\
\text { medicinal plants in a Caatinga area in } \\
\text { Ceará, northeastern Brazil }\end{array}$ & $\begin{array}{c}\text { Anais Revista } \\
\text { Brasileira de Ciências }\end{array}$ & 2017 \\
\hline 31 & $\begin{array}{l}\text { DA COSTA } \\
\text { et al. }\end{array}$ & $\begin{array}{c}\text { Ethnobotanical survey of medicinal flora } \\
\text { in the rural community } \\
\text { Rio dos Couros, state of Mato Grosso, } \\
\text { Brazil }\end{array}$ & $\begin{array}{l}\text { Boletín } \\
\text { Latinoamericano y } \\
\text { del Caribe de Plantas } \\
\text { Medicinales y } \\
\text { aromáticas }\end{array}$ & 2017 \\
\hline 32 & $\begin{array}{l}\text { CAVALHEIRO; } \\
\text { GUARIM-NETO }\end{array}$ & $\begin{array}{l}\text { Ethnobotany and regional knowledge: } \\
\text { combining popular knowledge with } \\
\text { the biotechnological potential of plants in } \\
\text { the Aldeia Velha community, } \\
\text { Chapada dos Guimarães, Mato Grosso, } \\
\text { Brazil }\end{array}$ & $\begin{array}{l}\text { Boletín } \\
\text { Latinoamericano y } \\
\text { del Caribe de Plantas } \\
\text { Medicinales y } \\
\text { aromáticas }\end{array}$ & 2018 \\
\hline 33 & $\begin{array}{l}\text { MESQUITA; } \\
\text { TAVARES- } \\
\text { MARTIN }\end{array}$ & $\begin{array}{l}\text { Etnobotánica de plantas medicinales en la } \\
\text { comunidad de Caruarú, } \\
\text { Isla del Mosqueiro, Belém-PA, Brasil }\end{array}$ & $\begin{array}{l}\text { Boletín } \\
\text { Latinoamericano y } \\
\text { del Caribe de Plantas } \\
\text { Medicinales y } \\
\text { aromáticas }\end{array}$ & 2018 \\
\hline 34 & $\begin{array}{l}\text { DA SILVA } \\
\text { MIGUÉIS et al. }\end{array}$ & $\begin{array}{l}\text { Plants used by the rural community of } \\
\text { Bananal, Mato Grosso, Brazil: Aspects of } \\
\text { popular knowledge }\end{array}$ & PLoS One & 2019 \\
\hline
\end{tabular}


Tabela 2 - Descrição sobre as principais famílias botânicas, partes das plantas utilizadas, formas de preparo e indicações de uso popular para os 34 artigos que compuseram essa revisão integrativa sobre levantamentos etnobotânicos de plantas medicinais realizados no período de 2010 a 2019.

\begin{tabular}{|c|c|c|c|c|}
\hline Ordem & $\begin{array}{c}\text { Total de famílias botânicas } \\
\text { citadas e famílias } \\
\text { predominantes }\end{array}$ & $\begin{array}{l}\text { Partes da } \\
\text { planta }\end{array}$ & $\begin{array}{l}\text { Formas de } \\
\text { preparo }\end{array}$ & Usos populares \\
\hline 01 & $\begin{array}{c}58 \text { famílias } \\
\text { Anacardiaceae e Caesalpinaceae }\end{array}$ & $*$ & $*$ & $\begin{array}{c}\text { Afecções respiratórias, } \\
\text { doenças de pele, e inflamação }\end{array}$ \\
\hline 02 & $\begin{array}{c}23 \text { famílias } \\
\text { Asteraceae, Fabaceae e } \\
\text { Lamiaceae }\end{array}$ & $\begin{array}{l}\text { Raiz, folhas e } \\
\text { flores }\end{array}$ & Infusão & $\begin{array}{c}\text { Efeito calmante e afecções } \\
\text { digestivas }\end{array}$ \\
\hline 03 & $\begin{array}{c}41 \text { famílias } \\
\text { Lamiaceae, Asteraceae e } \\
\text { Solanaceae }\end{array}$ & Folhas & Decocção & $\begin{array}{l}\text { Afecções digestivas, } \\
\text { respiratórias e dor }\end{array}$ \\
\hline 04 & $\begin{array}{l}16 \text { famílias } \\
\text { Asteraceae e Myrtaceae }\end{array}$ & Folhas & $*$ & Inflamação e dor \\
\hline 05 & $\begin{array}{c}38 \text { famílias } \\
\text { (Não descrita a predominante) }\end{array}$ & $\begin{array}{l}\text { Cascas do } \\
\text { caule, folhas e } \\
\text { raízes }\end{array}$ & $\begin{array}{l}\text { Lambedor, } \\
\text { Xarope e chá }\end{array}$ & Inflamações em geral \\
\hline 06 & $\begin{array}{l}25 \text { famílias } \\
\text { Asteraceae }\end{array}$ & $*$ & Infusão (chá) & $\begin{array}{l}\text { Afecções renais, digestivas, } \\
\text { respiratórias, cicatrizante }\end{array}$ \\
\hline 07 & $\begin{array}{c}33 \text { famílias } \\
\text { Asteraceae e Lamiaceae }\end{array}$ & Folhas & Infusão & $\begin{array}{l}\text { Afecções digestivas, } \\
\text { respiratórias, geniturinárias, } \\
\text { calmante }\end{array}$ \\
\hline 08 & $\begin{array}{l}33 \text { famílias } \\
\text { Asteraceae e Lamiaceae }\end{array}$ & Folhas & Decocção & $\begin{array}{c}\text { Gripe e tosse, afecções } \\
\text { digestivas e respiratórias, dor } \\
\text { de cabeça }\end{array}$ \\
\hline 09 & $\begin{array}{l}14 \text { famílias } \\
\text { Asteraceae e Lamiaceae }\end{array}$ & Folhas & $\begin{array}{l}\text { Infusão e } \\
\text { decocção }\end{array}$ & Gripe, afecções digestivas \\
\hline 10 & $*$ & Folhas & $\begin{array}{l}\text { Infusão } \\
\text { (bochecho) }\end{array}$ & Inflamação \\
\hline 11 & $\begin{array}{c}30 \text { famílias } \\
\text { Fabaceae } \\
\text { Apocynaceae e Anacardiaceae }\end{array}$ & Folhas & $\begin{array}{l}\text { Infusão e } \\
\text { decocção }\end{array}$ & Inflamação \\
\hline 12 & $\begin{array}{c}58 \text { famílias } \\
\text { Fabaceae, Asteraceae e } \\
\text { Lamiaceae }\end{array}$ & Folhas & Chá & $\begin{array}{l}\text { Afecções digestivas, } \\
\text { respiratórias, doenças de pele }\end{array}$ \\
\hline 13 & $\begin{array}{c}59 \text { famílias } \\
\text { Asteraceae, Fabaceae e } \\
\text { Lamiaceae }\end{array}$ & Folhas & $\begin{array}{l}\text { Lambedores, } \\
\text { garrafadas e } \\
\text { pó }\end{array}$ & $\begin{array}{l}\text { Afecções respiratórias e } \\
\text { digestivas, diarreias }\end{array}$ \\
\hline
\end{tabular}




\begin{tabular}{|c|c|c|c|c|}
\hline 14 & $\begin{array}{c}27 \text { famílias } \\
\text { (Não descrita a predominante) }\end{array}$ & $\begin{array}{l}\text { Cascas e } \\
\text { folhas }\end{array}$ & $\begin{array}{l}\text { Decocção, } \\
\text { infusão e } \\
\text { garrafada }\end{array}$ & $\begin{array}{l}\text { Afecções digestivas e } \\
\text { respiratórias }\end{array}$ \\
\hline 15 & $\begin{array}{c}12 \text { famílias } \\
\text { (Não descrita a predominante) }\end{array}$ & $\begin{array}{l}\text { Folhas e } \\
\text { cascas }\end{array}$ & $\begin{array}{l}\text { Decocção e } \\
\text { infusão }\end{array}$ & Afecções metabólicas \\
\hline
\end{tabular}

16

17

35 famílias
Fabaceae, Euphorbiaceae,
Anacardiaceae

$*$

Imersão,

infusão e

decocção

29 famílias
Lamiaceae e Asteraceae

In natura, pomadas,

tinturas,

xaropes

18

19

61 famílias
Asteraceae e Lamiaceae

Lamiaceae

20

21

37 famílias
Lamiaceae

31 famílias

Fabaceae e Euphorbiaceae

\begin{tabular}{c|c}
\hline Folhas & Infusão \\
\hline Folhas & Infusão
\end{tabular}

Afecções digestivas, geniturinárias e respiratórias

Afecções digestivas e respiratórias
Afecções respiratórias, digestivas e geniturinárias

Afecções digestivas e respiratórias

\begin{tabular}{c|c|c}
\hline Folhas & Decocção & $\begin{array}{c}\text { Afecções digestivas e } \\
\text { cicatrizante }\end{array}$ \\
\hline $\begin{array}{c}\text { Raízes e } \\
\text { cascas }\end{array}$ & $\begin{array}{c}\text { Infusão, } \\
\text { decocção e } \\
\text { lambedor }\end{array}$ & $\begin{array}{c}\text { Afecções digestivas, } \\
\text { respiratórias e inflamação }\end{array}$ \\
\hline
\end{tabular}
lambedor

22

94 famílias
Asteraceae e Lamiaceae

Folha
$*$

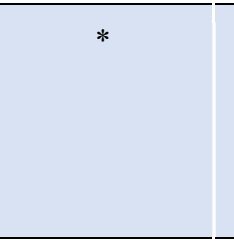

Via oral

60 famílias

Fabaceae

25

26

27

28

29

31 famílias
Fabaceae

Afecções digestivas, renais e respiratórias

Afecções cérebro-espinhais, respiratórias e digestivas

37 famílias

Fabaceae

20 famílias

\begin{tabular}{c}
\hline $\begin{array}{c}\text { Folhas e } \\
\text { cascas }\end{array}$ \\
Infusão \\
\hline
\end{tabular}

Chás,

lambedores e

garrafadas

Afecções digestivas e respiratórias

77 famílias

Asteraceae e Lamiaceae

Folhas Infusão

Inflamação em geral

\begin{tabular}{c|cc}
\hline Folhas & Infusão & $\begin{array}{c}\text { Afecções digestivas e } \\
\text { respiratórias }\end{array}$ \\
\hline Folhas & Infusão & Afecções digestivas
\end{tabular}


Acta Ambiental Catarinense - Unochapecó

\begin{tabular}{|c|c|c|c|c|}
\hline 30 & $\begin{array}{l}24 \text { famílias } \\
\text { Fabaceae e Euphorbiaceae }\end{array}$ & $\begin{array}{l}\text { Folhas e } \\
\text { raízes }\end{array}$ & $*$ & $\begin{array}{l}\text { Afecções digestivas e } \\
\text { respiratórias, cicatrizante de } \\
\text { ferimentos }\end{array}$ \\
\hline 31 & $\begin{array}{l}38 \text { famílias } \\
\text { Fabaceae }\end{array}$ & Folhas & Decocção & $\begin{array}{c}\text { Afecções respiratórias, renais } \\
\text { e digestivas. }\end{array}$ \\
\hline 32 & $\begin{array}{l}39 \text { famílias } \\
\text { Asteraceae }\end{array}$ & Folhas & Decocção & $\begin{array}{c}\text { Afecções respiratórias, renais } \\
\text { e digestivas }\end{array}$ \\
\hline 33 & $\begin{array}{l}33 \text { famílias } \\
\text { Fabaceae }\end{array}$ & Folhas & $\begin{array}{l}\text { Infusão e } \\
\text { decocção }\end{array}$ & Afecções geniturinárias \\
\hline 34 & $\begin{array}{c}67 \text { famílias } \\
\text { Asteraceae, } \\
\text { Fabaceae e Rutaceae }\end{array}$ & Folhas & Decocção & Afecções digestivas \\
\hline
\end{tabular}

*Não informado. 